\title{
Marcadores cardíacos na medicina veterinária
}

\author{
Cardiac markers in veterinary medicine
}

\author{
Letícia Andreza Yonezawa ${ }^{\mathrm{I}}$ Veridiana Fernandes da Silveira ${ }^{\mathrm{I}}$ \\ Luciana Pereira MachadoI Aguemi Kohayagawa ${ }^{\text {II }}$
}

\section{RESUMO}

Os marcadores cardíacos são utilizados com o intuito de auxiliar no diagnóstico clínico de animais com doença cardíaca com maior acurácia e em menor tempo possível, possibilitando o estabelecimento do prognóstico e a terapia precocemente. Entretanto, em medicina veterinária, no Brasil, sua aplicabilidade ainda é, em geral, restrita a pesquisas. Esta revisão tem como objetivo abordar os principais marcadores cardíacos para que futuramente estes possam se tornar índices essenciais na avaliação cardíaca.

Palavras-chave: lesão cardíaca, cardiomiopatia, medicina veterinária.

\section{ABSTRACT}

Cardiac markers have been used in order to assist clinical diagnosis of animals with heart disease, more accurately, enabling the establishment of earlier prognosis and therapy. However, in Brazilian veterinary medicine, these markers are generally still restricted to researches. This review aims to approach the major cardiac markers, which further may become essential indexes in heart assessment.

Key words: cardiac damage, cardiomyopathy, veterinary medicine.

\section{INTRODUÇÃO}

A identificação de animais com doença cardíaca assintomática por testes bioquímicos permite diagnosticar cardiopatas com maior acurácia e em menor tempo. Desse modo, é possível estabelecer um prognóstico e realizar a terapia precocemente (SISSON, 2002). A utilização dos marcadores cardíacos auxilia o clínico veterinário no diagnóstico de diversas cardiopatias, mas, para uma interpretação adequada dos resultados, são necessários exame físico detalhado e exames complementares, como o eletrocardiograma, a ecocardiografia, radiografia torácica e a mensuração da pressão arterial.

Os biomarcadores são indicadores de processos biológicos normais, processos patogênicos ou de intervenção farmacológica que fornecem informação considerando a exposição da doença, a extensão da lesão e o prognóstico (OYAMA \& SISSON, 2004). O marcador cardíaco ideal precisa atender a vários critérios, tais como: 1) alta sensibilidade, sendo detectado precocemente mesmo em baixa quantidade em injúria miocárdica leve; 2) alta especificidade, não sendo detectável em outros órgãos ou tecidos; 3 ) ser facilmente quantificado e a baixo custo; 4) apresentar boa janela diagnóstica, ou seja, aumentar mais precocemente, e o retorno aos níveis basais ser mais demorado (SHINDE et al., 2004; LADENSON, 2007). Atualmente, nenhum dos marcadores cardíacos disponíveis apresenta todos os critérios. Por isso,

IPrograma de Pós-graduação em Medicina Veterinária, área de concentração em Clínica Veterinária, Faculdade de Medicina Veterinária e Zootecnia (FMVZ), Universidade Estadual Paulista (Unesp), Campus de Botucatu. Distrito de Rubião Junior, s/n, 18618-000, Botucatu, SP, Brasil. E-mail: leticiay@gmail.com.*Autor para correspondência.

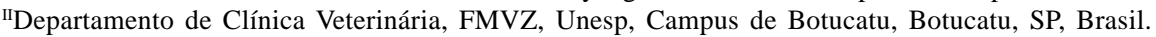


pesquisas devem ser realizadas em busca do preenchimento dessa lacuna.

O diagnóstico de injúria cardíaca em humanos a partir de biomarcadores iniciou-se por volta do ano de 1950. Atualmente, a literatura relacionada à área de marcadores cardíacos é bastante extensa, dificultando uma revisão completa sobre o assunto (LADENSON, 2007). Na medicina veterinária, muitos estudos clínicos sobre validação de testes ainda estão em andamento, e há evidências que sugerem que os benefícios potenciais destes são similares aos dos humanos (SOLTER, 2007).

Na medicina, os marcadores cardíacos incluem quatro tipos relacionados às seguintes condições clínicas: 1) lesão ou necrose miocárdica (troponinas cardíacas), 2) função miocárdica (peptídeos natriuréticos), 3) homeostase de lipoproteínas séricas (HDL, LDL), e 4) inflamação do sistema cardiovascular (proteína C reativa). Entretanto, em medicina veterinária, os dois últimos tipos de marcadores não são específicos para doença miocárdica (SOLTER, 2007). A presente revisão aborda os marcadores cardíacos mais utilizados em medicina veterinária, sua aplicabilidade e suas perspectivas futuras.

\section{Marcadores Cardíacos}

Peptídeos natriuréticos

Os peptídeos natriuréticos são importantes marcadores de hipertrofia ventricular e insuficiência cardíaca congestiva (ICC) (MAACK, 2006) e possuem valor, tanto no diagnóstico, como no prognóstico das cardiopatias (RICHARDS, 2007). O peptídeo natriurético atrial (ANP) e o peptídeo natriurético cerebral (BNP) são sintetizados a partir de precursores, proANP e proBNP, que são armazenados em grânulos do tecido atrial e em miócitos ventriculares, respectivamente. Os hormônios ANP e BNP ativos são clivados da porção carboxi-terminal das moléculas precursoras e secretados para a circulação com seus respectivos fragmentos amino-terminais (NT-proANP e NT-proBNP) (SISSON, 2004). O peptídeo natriurético tipo C (CNP) e seu peptídeo amino-terminal cosecretado foram recentemente identificados (SISSON, 2002; MAACK, 2006; RICHARDS, 2007). Esses peptídeos são encontrados primariamente no cérebro e endotélio vascular, e os níveis circulantes são muito menores em relação aos demais peptídeos, em animais hígidos (SISSON, 2004).

O ANP é o principal peptídeo circulante envolvido na regulação da função renal e do volume plasmático em condições fisiológicas. O aumento do volume plasmático e da pressão sanguínea arterial ou pulmonar leva à elevação significativa dos níveis de
ANP. O estímulo direto para a secreção de ANP é a distensão da parede ou pressão atrial, que ocorre na expansão volumétrica e hipertensão. Por outro lado, os níveis plasmáticos do BNP estão relacionados às condições de sobrecarga ventricular (MAACK, 2006; RICHARDS, 2007).

Os efeitos fisiológicos do ANP e BNP geralmente são opostos aos do sistema reninaangiotensina-aldosterona. Os peptídeos induzem, via receptor tipo A (NPR-A), a diurese e a natriurese pelo aumento da taxa de filtração glomerular e inibição do transporte do sódio do ducto coletor medular interno. O mesmo receptor regula a vasodilatação de arteríolas, diminuindo, assim, a resistência vascular sistêmica e pulmonar, a inibição direta da liberação de renina pelos rins e a liberação de aldosterona pelo córtex da adrenal. O ANP ainda reduz o débito cardíaco pela diminuição do volume plasmático e, consequentemente, a pressão venosa central. Um segundo receptor, o tipo B (NPR$\mathrm{B})$, responde ao ANP e BNP, mas regula a vasodilatação pelo CNP produzido no local (SISSON, 2004; MAACK, 2006).

A estrutura química do ANP é altamente preservada nas espécies de mamíferos. Por isso, a dosagem de ANP pode ser realizada por imunoensaios desenvolvidos para humanos (SISSON, 2004). Em contrapartida, o BNP apresenta considerável espécieespecificidade (MAACK, 2006). Recentemente, foram validados testes de ELISA (ensaio imunoabsorvente ligado à enzima) para determinação de NT-proANP e NT-proBNP em cães (BOSWOOD et al., 2008; SCHELLENBERG et al., 2008; TARNOW et al., 2009) e gatos (CONNOLLY et al., 2008). Preconiza-se a dosagem das porções amino-terminais, uma vez que possuem meia-vida mais prolongada em relação ao ANP e BNP, facilitando o diagnóstico de cardiopatias (OYAMA, 2007).

Muitos estudos foram conduzidos para avaliar a utilidade desses testes em cães, especialmente o NT-proBNP. Este parece diferenciar cães com doença cardíaca dos hígidos, além de determinar quais animais possuem aumento cardíaco significativo (BOSWOOD et al., 2008; OYAMA et al., 2008). Além disso, juntamente com o NT-proANP, auxiliam no diagnóstico diferencial de causas cardíacas e não cardíacas de distúrbios respiratórios em cães. Níveis muito elevados (NTproANP, >1.700fmol mL $\mathrm{fl}^{-1}$; NT-proBNP, >1.000 $\mathrm{pmol} \mathrm{L}^{-1}$ ) sugerem a ICC como causa da dispneia; níveis moderados (NT-proANP, 1.351-1.700fmol mL $\mathrm{fm}^{-1}$; NTproBNP, > 210pmol L ${ }^{-1}$ ) são sugestivos de cardiopatia, mas não necessariamente ICC em animais assintomáticos. Esses achados sugerem que a utilização desses testes são uma ferramenta útil no 
diagnóstico de doenças cardíacas assintomáticas ou ocultas (OYAMA, 2007).

O BNP também avalia a severidade da injúria isquêmica, uma vez que a isquemia causa aumento no estresse da parede ventricular esquerda, levando ao acréscimo da produção e à liberação de BNP, mesmo na ausência de necrose ou disfunção ventricular préexistentes (FUTTERMAN \& LAMBERG, 2002). Além disso, esse peptídeo também é útil no monitoramento de terapias que potencialmente podem causar cardiotoxicidade, como a doxorrubicina (ALVES DE SOUZA \& CAMACHO, 2006).

Duas condições que podem confundir parcialmente a utilidade do diagnóstico ou prognóstico das mensurações desses peptídeos são a disfunção renal e a presença da síndrome metabólica (RICHARDS, 2007; REYNOLDS \& OYAMA, 2008). Outro estudo demonstra também que, como em humanos, anticorpos heterofílicos podem interferir em teste de ELISA para BNP canino, ocasionando resultados falso-positivos (SOLTER et al., 2008).

Atualmente, as pesquisas estão sendo conduzidas para determinar se o NT-proBNP pode ser usado para monitoramento seriado de cães com doença assintomática da valva mitral, para avaliar o risco individual de desenvolvimento da insuficiência cardíaca secundária (REYNOLDS, \& OYAMA, 2008).

\section{Creatinoquinase}

A creatinoquinase (CK) é uma molécula constituída por duas subunidades, $\mathrm{M}$ e $\mathrm{B}$, que são imunologicamente distintas e sintetizadas por genes diferentes (AKTAS et al., 1993; KRAMER \& HOFFMANN, 1997). Possui a função de tornar adenosina trifosfato (ATP) disponível para a contração muscular, por meio da fosforilação de adenosina difosfato (ADP) e creatina fosfato. São três as isoenzimas formadas: CK-BB (CK1), predominante no cérebro e sistema digestório; CK-MB (CK2), presente no tecido cardíaco, nos rins, nos intestinos e nos pulmões; e CK-MM (CK3), presente no músculo estriado (AKTAS et al., 1993).

A determinação da CK pode ser realizada no soro ou plasma heparinizado, e a hemólise e a hiperbilirrubinemia podem resultar em falsos aumentos (AKTAS et al., 1993). Pode-se mensurar, tanto por sua atividade sérica por meio de testes bioquímicos, como por sua concentração total (CK massa), utilizando ensaios imunométricos. As isoenzimas podem ser separadas por eletroforese, técnicas imunológicas e cromatografia de troca iônica. Os métodos imunológicos requerem anticorpos monoclonais espécie-específicos (CARDINET III, 1997). No entanto,
SANTOS (2005) demonstrou a viabilidade da utilização de kit comercial imunométrico desenvolvido para humanos em cães.

O aumento da atividade sérica da CK-MB foi relatado em cães com comprometimento miocárdico secundário à parvovirose, dirofilariose, endocardite e cardiomiopatia hipertrófica (AKTAS et al., 1993). Em cães com suspeita de lesão miocárdica provocada por trauma, SCHOBER et al. (1999) e DINIZ et al. (2007) observaram aumento da CK-MB. Porém, DINIZ et al. (2007) acreditam que houve liberação de CK-MB, tanto de miocárdio, como de músculo esquelético, pois os animais apresentavam lesão muscular concomitante. PINO et al. (2008) encontraram níveis de CK-MB significativamente maiores em cães com algum tipo de doença cardiovascular quando comparados a cães hígidos. Segundo os autores, a hipóxia instalada ocasiona instabilidade e altera a permeabilidade da membrana dos miócitos, acarretando a elevação da enzima no soro. FRANCO et al. (2009) avaliaram cadelas anestesiadas com cetamina, atropina e xilazina e observaram aumento de CK-MB, sugerindo que os anestésicos reduzem o aporte de oxigênio do miocárdio.

ARGIROUDIS et al. (1982) analisaram o soro e os tecidos de equinos quanto à porcentagem de composição das isoenzimas da CK. Observaram quantidade de CK-MB muito pequena presente no coração $(1,4 \%$ da $C K$ total) e sugeriram que a determinação da isoenzima MB no soro de equinos não tem valor na detecção de lesão cardíaca. FREDERICK et al. (2001) consideram que a CK-MB não seja um marcador cardíaco adequado em cães, por ser pouco específico para essa espécie, além de representar de 4 a $13 \%$ da atividade total da CK no miocárdio canino (SCHOBER et al., 1999).

Devido a limitações, como a baixa especificidade nos casos de acometimento muscular esquelético associado, e à baixa sensibilidade nas primeiras horas de evolução em razão do aparecimento demorado desses marcadores no sangue e para detectar pequena injúria miocárdica, houve a necessidade de outros métodos ou novos marcadores diagnósticos de lesão celular miocárdica, visando a superar as limitações (GODOY et al., 1998).

Troponinas cardíacas

As troponinas vêm recebendo atenção crescente como marcadores altamente específicos de injúria celular (GODOY et al., 1998) e atualmente são consideradas como os preferíveis de injúria cardíaca em mamíferos (O’BRIEN, 2008). A estrutura dessas troponinas é muito parecida entre as espécies (O’BRIEN et al., 1997). 
Compreendem um complexo proteico que forma parte do aparato contrátil, o complexo troponinatropomiosina, encontrado dentro dos sarcômeros dos músculos estriados. Esse complexo age na regulação da contração muscular e é composto pela tropomiosina e por três troponinas: troponina C (TnC), I (TnI) e T (TnT) (COLLINSON et al., 2001).

A TnI (subunidade inibitória), quando ligada à actina, age em conjunto com a tropomiosina, criando um obstáculo espacial e inibindo a interação da actina com a miosina. A TnC é a subunidade que se liga ao cálcio, e a TnT (subunidade estrutural) liga o complexo troponina à tropomiosina. Na despolarização do sarcolema, há liberação de cálcio no citoplasma, e a TnC remove os efeitos inibitórios da TnI. Isso faz com que a tropomiosina se desloque, resultando na remoção do obstáculo espacial da interação da actina-miosina e liberação da inibição da actina-miosina-ATPase, permitindo a hidrólise do ATP e a contração muscular. Quando o cálcio é bombeado de volta para o retículo sarcoplasmático, o complexo reverte para sua conformação original, inibindo a ação da ATPase e ocasionando o relaxamento muscular (COLLINSON et al., 2001; O’BRIEN et al., 2008).

A TnC é idêntica, tanto no músculo esquelético, como no cardíaco. No entanto, os genes codificadores das TnT e TnI, cardíaca e esquelética, são diferentes, além de apresentarem sequências de aminoácidos distintas, o que permitiu que anticorpos monoclonais específicos pudessem ser desenvolvidos (GODOY et al., 1998). Em contraste à troponina I cardíaca (cTnI), a troponina T cardíaca (cTnT) não é totalmente cardio-específica. Esta é expressa no músculo em regeneração, assim como no músculo esquelético normal, e sua concentração sanguínea pode aumentar na ausência de envolvimento miocárdico, em pacientes com polimiosite, rabdomiólise, doença muscular crônica e insuficiência renal (SHINDE et al., 2004).

A liberação de cTnI na circulação parece estar relacionada às áreas de necrose de cardiomiócitos de maneira irreversível, sendo o aumento da concentração proporcional à severidade da injúria. A maior parte da cTnI é encontrada no aparato contrátil e é liberada por degradação proteolítica. O rompimento das proteínas contráteis intracelulares como resultado de isquemia ocorre rapidamente, causando um pool citosólico (COLLINSON et al., 2001). A cTnI é liberada em três formas: livre, complexo TnI-TnC e complexo TnT-TnI-TnC, que apresentam diferentes graus de degradação, e a parte predominante de cTnI circula na forma de complexo (OYAMA\& SOLTER, 2004).
Outro mecanismo de liberação é explicado pelo aumento da permeabilidade da membrana dos miócitos ocasionado por hipóxia, permitindo que somente a fração citosólica seja liberada de maneira reversível (MAIR, 1999; COLLINSON et al., 2001). Em cães, essa fração de troponina livre no citosol corresponde de 2 a $8 \%$ do total presente no cardiomiócito (SCHOBER et al., 2002), mas em equinos o significado desse pool é desconhecido (HOLBROOK et al., 2006).

KATRUKHA et al. (1997) mostraram que, em tecido cardíaco necrótico e no soro de pacientes que sofreram infarto agudo do miocárdio, as regiões amino- e carboxi-terminal da cTnI são vulneráveis à degradação. Em outro estudo, SHI et al. (1999) demonstraram que a região amino-terminal parece ser mais estável que a carboxi-terminal. Esses achados são importantes para explicar a ampla variação dos valores mensurados por diferentes ensaios de cTnI (SHINDE et al., 2004).

Os imunoensaios atuais podem mensurar elevadas concentrações de cTnI no sangue periférico, reflexo de lesões mínimas no miocárdio, mesmo na ausência de níveis aumentados de CK ou CK-MB (GUPTA \& DE LEMOS, 2007). Em ovinos induzidos experimentalmente ao infarto de miocárdio, observouse aumento de cTnI no primeiro dia após a indução e a concentração retornou ao nível normal após 14 dias (LEONARDI et al., 2008). Em humanos, a cTnI pode ser detectada cerca de 4 a 6 h após o início da isquemia, com pico em aproximadamente 24h, permanecendo elevada por pelo menos cinco dias após o episódio de infarto do miocárdio. Apesar de sua meia-vida biológica ser de 120 minutos, possui boa janela de diagnóstico em razão da contínua liberação dessa proteína dos miofilamentos em desintegração (SHINDE et al., 2004; GUPTA \& DE LEMOS, 2007). No entanto, em cães, JAFFE et al. (1996), ao injetar troponina purificada, observaram tempo de meia-vida de apenas 67,2 minutos.

As troponinas cardíacas vêm se tornando alvo de pesquisas na medicina veterinária nos últimos anos. Os ensaios desenvolvidos para dosagem de troponina humana já foram validados para várias espécies animais (OYAMA\& SOLTER, 2004; SANTOS, 2005; MICHIMA, 2007; LEONARDI et al., 2008). O aumento de seus níveis foi descrito em cães com cardiomiopatia dilatada (OYAMA \& SISSON, 2004), trauma cardíaco (SCHOBER et al., 1999; DINIZ et al., 2007), infarto (RICCHIUTI et al., 1998), degeneração da valva mitral (OYAMA \& SISSON, 2004), ICC (DEFRANCESCO et al., 2002), além de outras enfermidades que causam lesão cardíaca secundária, como síndrome dilatação vôlvulo-gástrica (SCHOBER 
et al., 2002) e babesiose (LOBETTI et al., 2002). Em gatos, níveis elevados de cTnI foram descritos em casos de cardiomiopatia hipertrófica (HERNDON et al., 2002) e em equinos, em lesão por ruptura da válvula aórtica (CORNELISSE et al., 2000), taquicardia ventricular e necrose miocárdica (SCHWARZWALD et al., 2003), sepse neonatal (SLACK et al., 2005), babesiose (DIANA et al., 2007), intoxicação por monensina e cardiopatia (PEEK et al., 2004).

Em equinos, a dosagem sérica de cTnI também é utilizada para avaliar o efeito do exercício físico. DURANDO et al. (2006), avaliando o exercício intenso de curta duração em esteira de alta velocidade, verificaram que as concentrações máximas de cTnI ocorrem entre 3 e 6 h após o exercício. Esse período parece ser ótimo para mensurar a cTnI com o intuito de detectar lesão miocárdica induzida pelo exercício em equinos. YONEZAWA(2008) observou lesão cardíaca leve em equinos Puro Sangue Árabe submetidos a exercício em esteira de alta velocidade, sendo verificada pela presença de contração ventricular prematura associada ao aumento de cTnI sérica, porém não expressiva. MICHIMA (2007) avaliou cavalos de enduro que percorreram provas menores que $100 \mathrm{~km} \mathrm{e}$ maiores que $100 \mathrm{~km}$ e animais desqualificados das provas por causa metabólica, não encontrando aumento significativo dos níveis de cTnI e concluindo que o exercício físico prolongado não levou a injúrias cardíacas severas.

Embora o mecanismo ainda não tenha sido totalmente elucidado, especula-se que o aumento pósexercício seja devido ao aumento da permeabilidade da membrana celular do miocárdio e à liberação de cTnI livre do citosol e não devido à necrose permanente e morte celular (NOSTELL \& HÄGGSTRÖM, 2008). Equinos que realizam teste de exercício máximo tornamse hipoxêmicos e possuem maior estresse cardiovascular, por isso apresentam leve aumento da cTnI (DURANDO et al., 2006).

Novos marcadores cardíacos

Muitas outras macromoléculas parecem aumentar nos distúrbios cardíacos em humanos e apresentam potencial para atuarem como novos possíveis marcadores cardíacos. No entanto, ainda não se sabe totalmente seu valor diagnóstico. Alguns destes serão descritos a seguir.

\section{Proteína ligadora de ácido graxo}

A proteína ligadora de ácido graxo tipo cardíaca (H-FABP) tem o papel de transporte intracelular citoplasmático de ácidos graxos nas células e é altamente expressa em tecidos com metabolismo ativo de ácido graxo, como o músculo cardíaco (RUZGAR et al., 2006). Compreende de 15 a 30\% de todas as proteínas citoplasmáticas (GODOY et al., 1998) e assemelha-se à mioglobina em relação ao baixo peso molecular e dinâmica do aparecimento na circulação, mas apresenta maior especificidade e sensibilidade. Entretanto, não é específica para lesões cardíacas, podendo ser encontrada no músculo esquelético, nos rins e em outros tecidos, em menores quantidades (SHINDE et al., 2004; RUZGAR et al., 2006).

Após a lesão causada pela isquemia, a HFABP é liberada rapidamente para a circulação e pode ser detectada em altas concentrações dentro de $1 \mathrm{~h}$ após a necrose miocárdica, podendo alcançar o máximo no período de 6 a 8 h e diminuindo em 24 a 36h (CHAN et al., 2004; RUZGAR et al., 2006). A curva de liberação da H-FABP demonstra que é um marcador precoce, mas perde o significado como marcador tardio em razão de sua rápida depuração renal (CHAN et al., 2004).

Os métodos comumente utilizados para a dosagem de H-FABP são os imunoensaios. Se a HFABP for mensurada conjuntamente com as troponinas cardíacas, podem formar um perfil bioquímico completo de detecção de injúria cardíaca, desde os estágios precoces até os mais tardios da doença. No entanto, o período de dosagem necessário para o "painel cardíaco” ainda é muito longo para ser usado na emergência (MION et al., 2007).

\section{Albumina modificada pela isquemia}

Os marcadores cardíacos disponíveis atualmente apresentam limitada sensibilidade em casos de condição isquêmica reversível no início da lesão. A albumina modificada pela isquemia (IMA) foi relatada como marcador capaz de refletir a condição isquêmica do miocárdio. A porção amino-terminal da albumina plasmática normalmente tem afinidade com metais pesados iônicos, como o cobalto e o cobre, e a porção N-terminal é modificada durante a exposição à condição isquêmica, em razão da produção de radicais livres e de espécies reativas ao oxigênio, resultando na geração de IMA com baixa afinidade aos metais pesados (LEE et al., 2007). A mensuração da IMA é realizada indiretamente pelo método de ligação do cobalto à albumina, e uma quantidade de cobalto constante reage com o soro do paciente e se liga à albumina, enquanto que o cobalto não ligado remanescente é mensurado por espectrofotometria (APARCI et al., 2007; LEE et al., 2007).

Há relatos que a IMA aumenta dentro de alguns minutos após o início da isquemia miocárdica e que continua a aumentar por 6 a $12 \mathrm{~h}$, sugerindo que pode ser aplicada efetivamente para a detecção de 
condição de isquemia miocárdica anterior à necrose miocárdica (CHRISTENSON et al., 2001). LEE et al. (2007) demonstraram que a IMA foi suficientemente sensível como marcador de triagem capaz de determinar se pacientes com dor torácica e suspeita de síndromes coronarianas agudas que necessitavam de terapia intensiva ou tratamento de emergência. Observou-se que a sensibilidade foi maior em relação aos demais marcadores cardíacos (mioglobina, CK-MB e cTnT), provavelmente porque a IMA eleva-se em condições de isquemia anteriores ao desenvolvimento da necrose tecidual. No entanto, a especificidade foi menor em relação aos demais, pois a IMA não reflete somente em isquemia cardíaca, mas também em outras condições isquêmicas, como no cérebro, no trato gastrintestinal e nos outros órgãos, assim como em outras doenças. Portanto, sugere-se que a dosagem de IMA deva ser realizada em paralelo aos marcadores convencionais, para compensar sua baixa especificidade e distinguir entre isquemia e necrose miocárdica.

\section{CONCLUSÕES}

Embora os marcadores cardíacos sejam amplamente utilizados na rotina clínica médica e na medicina veterinária em diversos países, no Brasil, o uso desses testes é restrito a pesquisas. Apesar do crescente número de estudos clínicos e da padronização dos métodos de dosagem, ainda há limitações, como disponibilidade e custo elevado das determinações para o uso ambulatorial. Espera-se que esses testes se tornem mais acessíves a fim do amplo uso na rotina clínica veterinária, tanto para o diagnóstico das cardiopatias, como para o monitoramento de terapias e estabelecimento de prognóstico, visando a uma melhor qualidade de vida dos pacientes.

\section{REFERÊNCIAS}

AKTAS, D.M. et al. Creatine kinase in the dog: a review. Veterinary Research Communications, v.17, n.5, p.353369, 1993.

ALVES DE SOUZA, R.C.; CAMACHO, A.A. Neurohormonal, hemodynamic, and electrocardiographic evaluations of healthy dogs receiving long-term administration of doxorubicin. American Journal of Veterinary Research, v.67, n.8, p.1319-1325, 2006.

APARCI, M. et al. Prognostic significance of ischemia-modified albumin in patients with acute coronary syndrome. Coronary Artery Disease, v.18, n.5, p.367-373, 2007.

ARGIROUDIS, S.A. et al. Observations on the isoenzymes of creatine kinase in equine serum and tissues. Equine Veterinary Journal, v.14, n.4, p.317-321, 1982.
BOSWOOD, A. et al. The diagnostic accuracy of different natriuretic peptides in the investigation of canine disease. Journal of Small Animal Practice, v.49, n.1, p.26-32, 2008. Disponível em: <http://www3.interscience.wiley.com/cgi-bin/ fulltext/119393985/PDFSTART>. Acesso em: 10 mar. 2009. doi: 10.1111/j.1748-5827.2007.00510.x.

CARDINET III, G.H. Skeletal muscle function. In: KANEKO, J.J. et al. Clinical Biochemistry of Domestic Animals. San Diego: Academic, 1997. p.407-440.

CHAN, C.P.Y. et al. A superior early myocardial infarction marker. Human heart-type fatty acid-binding protein. Zeitschrift für Kardiologie, v.93, n.5, p.388-397, 2004. Disponível em: <http://www.springerlink.com/content/ rwn6kd14qmfpuw4g/fulltext.pdf >. Acesso em: 24 set. 2007. doi: 10.1007/s00392-004-0080-6.

CHRISTENSON, R.H. et al. Characteristics of an albumin cobalt binding test for assessment of acute coronary syndrome patients: a multicenter study. Clinical Chemistry, v.47, n.3, p.464-470, 2001. Disponível em: <http://www.clinchem.org/ cgi/reprint/47/3/464>. Acesso em: 24 set. 2007.

COLLINSON, P.O. et al. Measurement of cardiac troponins. Annals of Clinical Biochemistry, v.38, n.5, p.423-449, 2001.

CONNOLLY, D.J. et al. Circulating natriuretic peptides in cats with heart disease. Journal of Veterinary Internal Medicine, v.22, n.1, p.96-105, 2008.

CORNELISSE, C.J. et al. Concentration of cardiac troponin I in a horse with a ruptured aortic regurgitation jet lesion and ventricular tachycardia. Journal of the American Veterinary Medical Association, v.217, n.2, p.231-235, 2000.

DEFRANCESCO, T.C. et al. Prospective clinical evaluation of serum cardiac troponin $\mathrm{T}$ in dogs admitted to a veterinary teaching hospital. Journal of Veterinary Internal Medicine, v.16, n.5, p.553-557, 2002.

DIANA, A. et al. Cardiac arrhythmias associated with piroplasmosis in the horse: a case report. Veterinary Journal, v.174, n.1, p.193-195, 2007. Disponível em: <http://www.sciencedirect.com/ science?_ob=ArticleURL\&_udi=B6WXN-4K5JBY 8$18 \_$user=972052\&_rdoc $=1 \& \_$fmt $=$\&_orig=search\&_sort=d\&_docanch or $=\&$ view $=$ c\&_acct $=C 000049647$ \&_version $=1 \&$ \&urlVers ion $=0 \& \_$userid $=972052 \& m d 5=7 f 7 d 300257472 d 972 d b f 915 c 2 c 3 d 8 d 10>$. Acesso em 24 set. 2007.

DINIZ, P.P.V.P. et al. Cardiac trauma confirmed by cardiac markers in dogs: two case reports. Arquivos Brasileiros de Medicina Veterinária e Zootecnia, v.59, n.1, p.85-89, 2007. Disponível em: <http://www.scielo.br/scielo.php?script=sci_arttext\&pid=S010209352007000100015\&lng=pt\&nrm=iso\&tlng=en>. Acesso em: 03 out. 2007. doi: 10.1590/S0102-09352007000100015.

DURANDO, M.M. et al. Acute effects of short duration maximal exercise on cardiac troponin I in healthy horses. Equine and Comparative Exercise Physiology, v.3, n.4, p.217-223, 2006. Disponível em: <http://journals.cambridge.org/download.php?file=/ ECP/ECP3_04/S 1478061506703048 a.pdf\&code $=$ d23a50ad976fe2849ea5704c1f57a0b3>. Acesso em: 22 mai 2007. doi: $10.1017 / \mathrm{S} 1478061506703048$. 
FRANCO, L.G. et al. Assessment of serum enzymatic markers of cardiomyocytes injury in female dogs submitted to ketamine $\mathrm{S}(+)$, atropin and xylazine association. Acta Cirúrgica Brasileira, v.24, n.1, p.36-42, 2009. Disponível em: <http:/ /www.scielo.br/scielo.php?script=sci_arttext\&pid=S0102 $86502009000100008 \& \operatorname{lng}=p t \& n r m=i s o \& t \operatorname{lng}=e n>$. Acesso em: 10 mar. 2009. doi: 10.1590/S0102-86502009000100008.

FREDERICK, S. et al. Cardiac troponins and creatine kinase content of striated muscle in common laboratory animals. Clinica Chimica Acta, v.304, n.1-2, p.65-74, 2001. Disponível em: < h t t p : / / w w w. s c i e n c e d i r e c t. c o m science?_ob=ArticleURL\&_udi=B6T57-4281031$8 \& \_$user $=972052 \& \_$rdoc $=1 \& \_\mathrm{fmt}=\& \_$orig $=$search $\& \_$sort $=\mathrm{d} \&$ _docanchor $=\&$ view $=$ c\&_acct $=$ C000049647\&_version $=1$ \&_urlV e r si o n $=0$ \&_us e r i d $=972052 \&$ m d $5=$ 637aadf72d57d11707d4bb93a4ee1aa0>. Acesso em: 10 mar. 2009. doi: 10.1016/S0009-8981(00)00409-5.

FUTTERMAN, L.G.; LAMBERG, L. Novel markers in the acute coronary syndrome: BNP, IL-6, PAPP-A. American Journal of Critical Care, v.11, n.2, p.168-172, 2002. Disponível em: <http://ajcc.aacnjournals.org/cgi/content/full/ 11/2/168>. Acesso em: 24 set. 2007.

GODOY, M.F. et al. Troponina como marcador de injúria celular miocárdica. Arquivos Brasileiros de Cardiologia, v.71, n.4, p.629-633, 1998 . Disponível em: <http:// www.scielo.br/scielo.php?script=sci_arttext\&pid=S0066$782 X 1998001000013 \& \operatorname{lng}=p t \& n r m=i s o \& t l n g=p t>$. Acesso em: 24 set. 2007. doi: 10.1590/S0066-782X1998001000013.

GUPTA, S.; DE LEMOS, J.A. Use and misuse of cardiac troponins in clinical practice. Progress in Cardiovascular Diseases, v.20, n.2, p.151-165, 2007. Disponível em: <http://www.sciencedirect.com/ science?_ob=ArticleURL\&_udi=B75BG-4PJBPNG$6 \&$ _user $=972052 \&$ _rdoc $=1 \&$ \& fmt $=\&$ \&orig $=$ search \&_so $\mathrm{rt}=\mathrm{d} \&$ _docanchor $=\&$ view $=\mathrm{c} \&$ _acct $=\mathrm{C} 000049647 \&$ _ version $=1 \&$ _ u r l Version $=0 \&$ _ us erid $=97$ 2052\&md5=ae5f9024a0af1f0a354eae2e0aaa4f49>. Acesso em: 24 set 2007. doi: 10.1016/j.pcad.2007.01.002.

HERNDON, W.E. et al. Cardiac troponin I in feline hypertrophic cardiomyopathy. Journal of Veterinary Internal Medicine, v.16, n.5, p.558-564, 2002.

HOLBROOK, T.C. et al. Endurance exercise is associated with increased plasma cardiac troponin I in horses. Equine Veterinary Journal Supplement, v.36, p.27-31, 2006.

JAFFE, A.S. et al. Comparative sensitive of cardiac troponin I and lactate dehydrogenase isoenzymes for diagnosing acute myocardial infarction. Clinical Chemistry, v.42, n.11, p.1770-1776, 1996. Diponível em: <http://www.clinchem.org/ cgi/reprint/42/11/1770>. Acesso em: 24 set. 2007.

KATRUKHA, A.G. et al. Troponin I is released in bloodstream of patients with acute myocardial infarction not in free form but as a complex. Clinical Chemistry, v.43, n.8, p.13791385, 1997. Disponível em: <http://www.clinchem.org/cgi/ content/full/43/8/1379>. Acesso em: 24 set. 2007.

KRAMER, J.W.; HOFFMANN, W.E. Clinical enzimology. In: KANEKO, J.J. et al. Clinical biochemistry of domestic animals. San Diego: Academic, 1997. p.303-325.
LADENSON, J.H. A personal history of markers of myocyte injury [myocardial infarction]. Clinical Chimica Acta, v.381, n.1, p.3-8, 2007. Disponível em: <http://www.sciencedirect.com/ science? ob=ArticleURL \& udi=B6T57-4N56BV2 $1 \& \_$user $=972052 \& \_$rdoc $=18 \_\mathrm{fmt}=$ \&_orig $=$ search\&_sort $=\mathrm{d} \&$ _docanchor $=\&$ view $=$ c \&_acct $=$ C $000049647 \&$ \& ve rsion $=1 \& \quad$ url Version $=0 \& \quad$ use rid $=97205$ 2\&md5=f929e5ed5f64cc2b7e8a622c56ba10b4>. Acesso em: 24 set. 2007. doi: 10.1016/j.cca.2007.02.039.

LEE, Y.W. et al. Application of albumin-adjusted ischemia modified albumin index as an early screening marker for acute coronary syndrome. Clinical Chimica Acta, v.384, n.1-2, p.24-27, 2007. Disponível em: <http://www.sciencedirect.com/ science?_ob=ArticleURL\&_udi=B6T57-4NS0KWV$2 \& \_u s e r=972052 \& \_r d o c=1 \& \_f m t=\& \_o r i g=$ search \&_so $\mathrm{rt}=\mathrm{d} \&$ \&docanchor $=\& \mathrm{view}=\mathrm{c} \&$ acct $=\mathrm{C} 000049647 \&$ \&version $=1 \&$ \& urlversion $=0 \&$ \&_userid $=972052 \&$ m d $5=$ e56fcf21261980639f6c72ebecde7823>. Acesso em: 24 set. 2007. doi: 10.1016/j.cca.2007.05.003.

LEONARDI, F. et al. Cardiac troponin I (cTnI) concentration in an ovine model of myocardial ischemia. Research in Veterinary Science, v.85, n.1, p.141-144, 2008. Disponível em: <http:// www.sciencedirect.com/science? ob=ArticleURL\& udi=B6WWR4R05BW3-2\&_user $=972052 \&$ \&doc $=1 \&$ fmt $=\&$ \&_orig $=$ search $\&$ s ort $=$ d \&_doc anchor $=\&$ view $=$ c \& _ a c c t $=$ C 000049647 \&_version $=1 \&$ \&urlVersion $=0 \&$ \&_userid $=97$ 2052\&md5=50f2a36e410d1cbdcac9538e8c4c9c2e>. Acesso em: 13 ago. 2008. doi: 10.1016/j.rvsc.2007.09.010.

LOBETTI, R. et al. Cardiac troponins in canine babesiosis. Journal of Veterinary Internal Medicine, v.16, n.1, p.6368, 2002.

MAACK, T. The broad homeostatic role of natriuretic peptides. Arquivos Brasileiros de Endocrinologia \& Metabologia, v.50, n.2, p.198-207, 2006. Disponível em: <http:// www.scielo.br/scielo.php?script=sci_arttext\&pid=S0004$27302006000200006 \& \operatorname{lng}=p t \& n r m=i s o \& t \operatorname{lng}=$ en $>$. Acesso em: 03 out. 2007. doi: 10.1590/S0004-27302006000200006.

MAIR, J. Tissue release of cardiac markers: from physiology to clinical applications. Clinical Chemistry and Laboratory Medicine, v.37, n.11-12, p.1077-1084, 1999. Disponível em: <http://www.reference-global.com/doi/abs/10.1515/ CCLM.1999.157>. Acesso em: 18 out. 2007. doi: 10.1515/ CCLM.1999.157.

MICHIMA, L.E.S. Influência do exercício físico prolongado sobre a concentração sérica de troponina I cardíaca e sobre a função cardíaca em cavalos de enduro. 2007. 94f. Tese (Doutorado em Clínica Veterinária) - Curso de Pós-graduação em Medicina Veterinária, Faculdade de Medicina Veterinária e Zootecnia, Universidade de São Paulo, SP.

MION, M.M. et al. Analytical and clinical performance of a fully automated cardiac multi-markers strategy based on protein biochip microarray technology. Clinical Biochemistry, v.40, n.16-17, p.1245-1251, 2007. Disponível em: <http://www.sciencedirect.com/ science?_ob=ArticleURL\&_udi=B6TDD-4PCXGJYB \& user $=972052 \&$ rdoc $=1 \&$ fmt $=\&$ orig $=$ search $\&$ _sort $=$ d\&_docanchor $=\&$ view $=$ c $\&$ _acct $=$ C 000049647 $\&$ version $=1 \&$ urlVersion $=0 \&$ userid $=972052 \& \mathrm{~m}$ d5=6f344d19a2068df8db739434f6616ab3 >. Acesso em: 03 out. 2007. doi: 10.1016/j.clinbiochem.2007.07.018. 
NOSTELL, K.; HÄGGSTRÖM, J. Resting concentrations of cardiac troponin I in fit horses and effect of racing. Journal of Veterinary Cardiology, v.10, p.105-109, 2008. Disponível em: $<\mathrm{h} \mathrm{t} \mathrm{t} \mathrm{p} \mathrm{:} \mathrm{/} \mathrm{/} \mathrm{w} \mathrm{w} \mathrm{w.} \mathrm{s} \mathrm{c} \mathrm{i} \mathrm{e} \mathrm{n} \mathrm{c} \mathrm{edire} \mathrm{c} \mathrm{t.} \mathrm{c} \mathrm{o} \mathrm{m/}$ science?_ob=MImg\&_imagekey=B7RN0-4TY919X-1$1 \&$ _cdi $=25764$ \&_user $=972052 \&$ _orig $=$ browse\&_coverDate $=12 / 31$ / $2008 \&$ \&k $=999899997 \& v i e w=c \& w c h p=d G L b V l W-$ zSkzk\&md5=a875a9ea7daa6bfdcc2bdda4923186fb\&ie=/sdarticle.pdf $>$. Acesso em: 10 mar. 2009. doi: 10.1016/j.jvc.2008.10.001.

O’BRIEN, P.J. Cardiac troponin is the most effective translational safety biomarker for myocardial injury in cardiotoxicity. Toxicology, v.245, p.206-218, 2008. Disponível em: <http:// www.sciencedirect.com/science?_ob=ArticleURL\&_udi=B6TCN4RCKKYM-2\&_user=972052\&_rdoc $=1 \& \_f m t=$ \&_orig $=$ search\&_s ort $=$ d\&_docanchor $=\&$ view $=c \& \_$acct $=C 000049647 \&$ _version $=1 \&$ \&urlVersion $=0 \&$ \&userid $=972052 \& \mathrm{~m}$ d5=7ba9d9a2cb533b393beaf498ae26e092>. Acesso em: 10 mar. 2009. doi: 10.1016/j.tox.2007.12.006.

O'BRIEN, P.J. et al. Differential reactivity of cardiac and skeletal muscle from various species in a cardiac troponin I immunoassay. Clinical Chemistry, v.43, n.12, p.2333-2338, 1997. Disponível em: <http://www.clinchem.org/cgi/content/ full/43/12/2333>. Acesso: 24 set. 2007.

OYAMA, M.A. Cardiac blood tests: when, how and why to use them. In: NORTH AMERICAN VETERINARY CONFERENCE, 2007, Orlando, USA. Proceedings... Orlando: NAVC, 2007. Online. Disponível em: <http://www.ivis.org/proceedings/navc/ 2007/SAE/058.asp?LA=1>. Acesso em: 07 jan. 2009.

OYAMA, M.A. et al. Clinical utility of serum N-terminal proB-type natriuretic peptide concentration for identifying cadiac disease in dogs and assessing disease severity. Journal of American Veterinary Medical Association, v.232, n.10, p.1496-1503, 2008.

OYAMA, M.A.; SISSON, D.D. Cardiac troponin-I concentration in dogs with cardiac disease. Journal of Veterinary Internal Medicine, v.18, n.6, p. 831-839, 2004.

OYAMA, M.A.; SOLTER, P.F. Validation of an immunoassay for measurement of canine cardiac troponin-I. Journal of Veterinary Cardiology, v.6, n.2, p.17-24, 2004. Disponível e $\mathrm{m}:<\mathrm{h} \mathrm{t} \mathrm{tp}: / / \mathrm{ww}$ w. scie n c edirect.com/ science?_ob=MImg\&_imagekey=B7RN0-4J8C7C3-31\&_cdi=25764\&_user=972052\&_orig=browse\&_coverDate=11/ $30 / 2004 \&$ sk $=999939997 \& v i e w=c \& w c h p=d G L z V t b-$ zSkWA\&md5=16863b69fb3b4be99e4505b2d8cba5d1\&ie=/ sdarticle.pdf $>$. Acesso em: 24 set. 2007.

PEEK, S.F. et al. Atypical acute monensin toxicosis and delayed cardiomyopathy in belgian draft horses. Journal of Veterinary Internal Medicine, v.18, n.5, p.761-764, 2004.

PINO, V.O. et al. Determinación de los niveles séricos de enzimas cardíacas em perros adultos com enfermedad cardiovascular. Revista de Investigaciones Veterinarias del Peru, v.19, n.2, p.144-147, 2008. Disponível em: <http:/ /sisbib.unmsm.edu.pe/bVrevistas/veterinaria/v19_n2/pdf/ a06v19n2.pdf>. Acesso em: 10 mar. 2009.

REYNOLDS, C.; OYAMA, M.A. Biomarkers in the diagnosis of canine heart disease. Veterinary Focus, v.18, n.3, p.2-6, 2008. Disponível em: <http://www.ivis.org/journals/vetfocus/ 18_3/en/1.pdf $>$. Acesso em: 07 jan. 2009.
RICCHIUTI, V. et al. Cardiac troponin I and T alterations in dog hearts with myocardial infarction: correlation with infarct size. American Journal of Clinical Pathology, v.110, n.2, p.241-247, 1998.

RICHARDS, A.M. Natriuretic peptides. Update on peptide release, bioactivity, and clinical use. Hypertension, v.50, n.1, p.25-30, 2007. Disponível em: <http://hyper.ahajournals.org/ cgi/reprint/50/1/25>. Acesso em: 24 set. 2007. doi: 10.1161/ HYPERTENSIONAHA.106.069153.

RUZGAR, O. et al. The use of human heart-type fatty acidbinding protein as an early diagnostic biochemical marker of myocardial necrosis in patients with acute coronary syndrome, and its comparison with troponin $\mathrm{T}$ and creatine kinasemyocardial band. Heart and Vessels, v.21, n.5, p.309-314, 2006. Disponível em: <http://www.springerlink.com/content/ g414321744715688/fulltext.pdf>. Acesso em: 24 set. 2007. doi: 10.1007/s00380-006-0908-2.

SANTOS, A.L.F. Dosagem sérica da enzima creatinafosfoquinase-isoenzima MB (CK-MB) de cães eletrocardiograficamente normais e naqueles com desníveis (infra e supra) do segmento ST, por meio de ensaio imunométrico por quimioluminescência. 2005. 61f. Dissertação (Mestrado em Clínica Veterinária) Curso de Pós-graduação em Medicina Veterinária, Faculdade de Medicina Veterinária e Zootecnia, Universidade de São Paulo, SP.

SCHELLENBERG, S. et al. Analytical validation of commercial immunoassays for the measurement of cardiovascular peptides in the dog. Veterinary Journal, v.178, p.85-90, 2008. Disponível em: $<\mathrm{h} \mathrm{t} \mathrm{t} \mathrm{p} \mathrm{:} \mathrm{/} \mathrm{/} \mathrm{w} \mathrm{w} \mathrm{w.} \mathrm{s} \mathrm{c} \mathrm{i} \mathrm{e} \mathrm{n} \mathrm{c} \mathrm{ed} \mathrm{i} \mathrm{re} \mathrm{c} \mathrm{t.} \mathrm{c} \mathrm{o} \mathrm{m} \mathrm{/}$ science?_ob=ArticleURL\&_udi=B6WXN-4PGH4KF$1 \&$ _user $=972052 \&$ \&doc $=1 \&$ \& f mt $=\&$ _orig $=$ search $\& \_$sort $=$d\&_docanchor $=\&$ view $=$ c $\&$ _acct $=$ C 0000 $49647 \&$ \& version $=1 \&$ \&_urlversion $=0 \&$ _userid $=$ 972052\&md5=67551de4eb1d4b8133f50e7dc2f2e0cc $>$. Acesso em: 10 mar. 2007. doi: 10.1016/j.tvjl.2007.07.002.

SCHOBER, K.E. et al. Noninvasive assessment of myocardial cell injury in dogs with suspected cardiac contusion. Journal of Veterinary Cardiology, v.1, n.2, p.17-25, 1999. Disponível em: $<\mathrm{h} \mathrm{t} \mathrm{t} \mathrm{p} \mathrm{:} \mathrm{/} \mathrm{/} \mathrm{w} \mathrm{w} \mathrm{w.} \mathrm{s} \mathrm{c} \mathrm{i} \mathrm{e} \mathrm{n} \mathrm{c} \mathrm{ed} \mathrm{i} \mathrm{re} \mathrm{c} \mathrm{t.} \mathrm{c} \mathrm{o} \mathrm{m/}$ science?_ob=MImg\&_imagekey=B7RN0-4J8C7C5-318_cdi=25764\&_user=972052\&_orig=browse\&_coverDate $=12 / 31$ / $1999 \& \_s k=999989997 \& v i e w=c \& w c h p=d G L b V l z-$ zSkWz\&md5=85ab20b5986fde1fcd69b98cb6615ad6\&ie =/ sdarticle.pdf>. Acesso em: 10 mar. 2007.

SCHOBER, K.E. et al. Serum cardiac troponin I and cardiac troponin $\mathrm{T}$ concentrations in dogs with gastric dilatationvolvulus. Journal of the American Veterinary Medical Association, v.221, n.3, p.381-388, 2002.

SCHWARZWALD, C.C. et al. High cardiac troponin I serum concentration in a horse with multiform ventricular tachycardia and myocardial necrosis. Journal of Veterinary Internal Medicine, v.17, n.3, p.364-368, 2003.

SHI, Q. et al. Degradation of cardiac troponin in serum complicates comparisons of cardiac troponin I assays. Clinical Chemistry, v.45, n.7, p.1018-1025, 1999. Disponível em: $<$ http://www.clinchem.org/cgi/content/full/45/7/1018>. Acesso em: 24 set. 2007. 
SHINDE, S.B. et al. Relevant cardiac biochemical markers. Bombay Hospital Journal, v.46, n.4, 2004. Disponível em: <http://www.bhj.org/journal/2004_4604_oct/oct\%202004/htm/ reveiw_articles_relevant.htm>. Acesso em 24 set. 2007.

SISSON, D. Biochemical markers of cardiac dysfunction. In: WORLD SMALL ANIMAL VETERINARY ASSOCIATION CONGRESS, 27., 2002, Granada, Spain. Proceedings... Granada: WSAVA, 2002. Disponível em: <http://www.vin.com/proceedings/ Proceedings.plx?CID=WSAVA2002\&PID $=2531 \&$ Category $=408>$. Acesso em 31 mar. 2006.

SISSON, D. Neuroendocrine evaluation of cardiac disease. Veterinary Clinics of North America. Small Animal Practice, v.34, n.5, p.1105-1126, 2004.

SLACK, J.A. et al. Biochemical markers of cardiac injury in normal, surviving septic, or nonsurviving septic neonatal foals. Journal of Veterinary Internal Medicine, v.19, n.4, p.577580, 2005.

SOLTER, P.F. Clinical biomarkers of cardiac injury and disease. In: ACVP/ASVCP CONCURRENT ANNUAL MEETINGS, 2007, Savannah, USA. Proceedings... Savannah: ACVP/
ASVCP, 2007. Disponível em: <http://www.ivis.org/proceedings/ acvp/2007/solter.pdf?LA=1>. Acesso em 19 fev. 2008.

SOLTER, P.F. et al. Canine heterophilic antibodies as a source of false-positive B-type natriuretic peptide sandwich ELISA results. Veterinary Clinical Pathology, v.37, n.1, p.86-95, 2008.

TARNOW, I. et al. Predictive value of natriuretic peptides in dogs with mitral valve disease. Veterinary Journal, v.180, p.1952001, 2009. Disponível em: <http://www.sciencedirect.com/ science? ob=ArticleURL \& udi=B6WXN-4T41PMN$1 \&$ _user $=972052 \&$ _rdoc $=1 \&$ \&mt $=\&$ \&orig $=$ search $\&$ _sort $=\mathrm{d} \&$ d o c a n c hor $=\&$ vi e w $=$ c \&_a c ct $=$ C 000049647 \& version $=1 \&$ urlversion $=0 \&$ userid $=972052$ $\& \overline{m d} 5=f 7 e 461195 a 97680601 \mathrm{e} 09 \mathrm{ba70dad4d6a}>$. Acesso em: 10 mar. 2009. doi: 10.1016/j.tvjl.2007.12.026

YONEZAWA, L.A. Malondialdeído, troponina I cardíaca e eletrocardiografia em equinos da raça Puro Sangue Árabe submetidos ao exercício e à suplementação com vitamina E (dl-alfa-tocoferol). 2008. 51f. Dissertação (Mestrado em Clínica Veterinária) - Curso de Pós-graduação em Medicina Veterinária, Faculdade de Medicina Veterinária e Zootecnia, Universidade Estadual Paulista, Campus de Botucatu, SP. 